\title{
Seamless Multimedia Service Mechanism Based on Content Profile Using User and Device ID for Personal Mobility in Smart Home
}

\author{
Wenquan $\mathrm{Jin}^{1}$ and DoHyeun $\mathrm{Kim}^{2 *}$ \\ ${ }^{1,2}$ Computer Engineering Department, Jeju National University, \\ Republic of Korea \\ ${ }^{1}$ wenquan.jin@jejunu.ac.kr, ${ }^{2}$ kimdh@jejunu.ac.kr
}

\begin{abstract}
Recently, there are available to build a smart home with advanced telecommunication technologies. We can use several network protocols for interoperability between devices in smart home. Through the ability of devices in smart homes, each device can be intelligent which involves computing, decision making or strategy making functionalities. The functionalities are also able to deploy in servers which may in a cloud. This paper presents a seamless multimedia service mechanism based on content profile using identifiers of users and devices in the smart home. We propose the personal mobility mechanism of multimedia contents in order to support multi-spaces and multi-devices and multi-users for seamless multimedia service. We consider the users, devices, locations and the profile of contents to support a mobility mechanism. The profile of contents are used to synchronize the state of contents on multi-devices for the mobility of users in the smart home. Additionally, we design and implement a seamless multimedia service mechanism with the identifiers of the users and devices, the profile of contents.
\end{abstract}

Keywords: Multimedia; Smart home; Personal Mobility; Multi-Device; Multi-Location

\section{Introduction}

Today, the life style is becoming smarter with technological advancements. The technologies render the life to be fun and the works to be easier and efficient. With the development of Internet of Things (IoT) technologies such as networks, sensors and computing power, heterogeneous electronic devices are deployed around us which involves as small as sensors or as large as automobiles. The technologies of artificial intelligence makes devices to be automatic and context aware. Smart things obviously have the ability to make life easier and more convenient. Smart environments have enabled the devices to be aware for the context such as human actions within the environment. Humans convey information through sounds and behaviors, and otherwise that we receive information through those to understand the situations in implicitly [1]. Therefore, the ability of conveying information does not transfer well for interaction between humans and computers. The familiar approach is that directly input explicit information to computers using keyboards or mice [2]. The novel machine learning and fuzzy solutions enable the implicit context to be improved [3] [4] [5]. The behavior of humans are described by finite number of states which from different sensors in the environment. In order to make smart and intelligent decisions, the system needs to collect and process more variety data which from the sensor networks.

In the smart home, users can enjoy the multimedia contents to spend leisure time. For accessing the contents in any time and any spaces in the home domain, the home should

Received (October 23, 2017), Review Result (December 19, 2017), Accepted (January 16, 2018)

* Corresponding Author 
be a system to support the intelligence service. In order to actively play some information to remind its users, these kinds of context information may include home situations, preferred settings of its residents, etc. Therefore, for smarter home service, Multimedia Service needs to involve recommendation and context awareness.

In this paper, the seamless multimedia service mechanism is proposed for the user mobility in smart homes. In order to support the seamless multimedia services to the user continuously in different locations, the information of user, device, location and the profile of contents are needed to be considered. Therefore, we present the analysis of seamless multimedia services for user mobility through sequence diagrams. Those sequence diagrams illustrate activities of interacting with the multimedia device in the smart home. According to the analysis from interactions of user, device, and server, we also present a data model using the ER-diagram which is used for implementing the DB in the server. Based on the analysis and consideration, the design and implementation of proposed mechanism's prototype are also presented to demonstrate the multimedia service mechanism for the user mobility in the smart home.

The rest of the paper is organized as follows. Section 2 introduces the multimedia services in smart homes. Section 3 introduces the analysis of multimedia contents in smart home. Section 4 describes the design of seamless multimedia IoT services for the personal mobility in smart home and Section 5 describes the implementation results. Finally, Section 6 concludes the paper.

\section{Related Work}

The smart home is one of the emerging application domains of IoT which collaborates heterogeneous technologies which enables to provide more comfortable user experience, safer and energy efficient functionalities. Several research groups are working on the application domain of smart homes [6]. Nearly 20 home labs are working on more than 30 appliances, over 5 network protocols, and more than 3 AI techniques from MIT, Siemens, Cisco, IBM, Xerox, Microsoft and etc. Therefore, the smart home is important in IoT paradigm. With the IoT is growing, smart things are around us. In home, electronic devices are deployed and replaced a lot of works for humans in the last decade [7]. However, sustained growing electronic device cannot provide more services [8]. Seamless and efficient collaboration between technologies can improve the abilities and also can generate new power of creativity. Smart homes obviously have the ability to make life easier and more efficient.

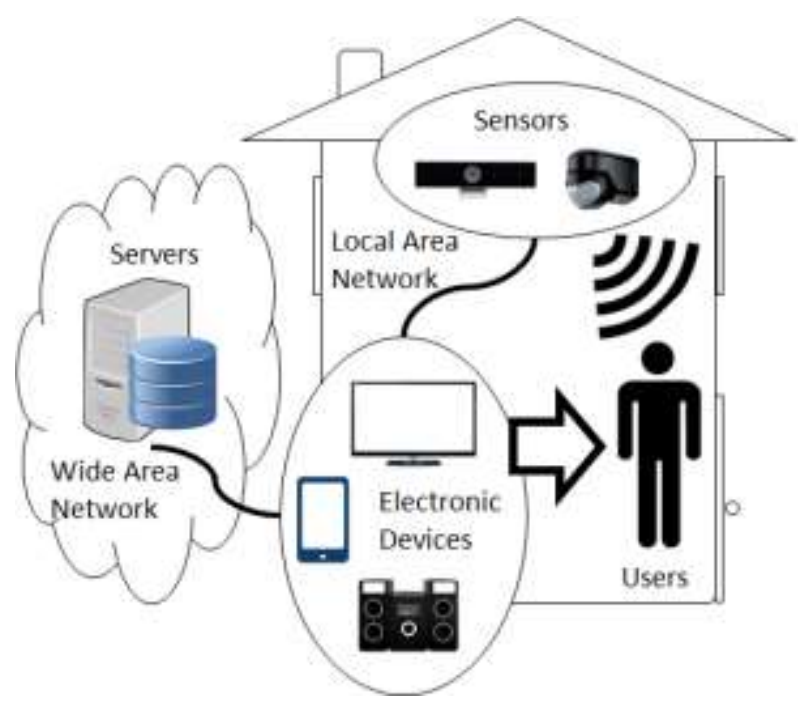

Figure 1. Multimedia Services in the Smart Home 
The smart home can automatically detect the changes of home environment attributes, dynamically make decisions for the corresponding reactions and autonomously support its users to be more comfortable [9]. The technologies of smart home are comprised by applications, home networks, and services. Those are supported by Information and Communication Technology (ICT) as well as a part of IoT. Through the interoperability of the elements, the home residents can easily enjoy home services such as multimedia services. However, which approaches can achieve living interactions between residents and smart home [10] [11]. These key issues can be accomplished by applying the context aware computing [12].

A smart home involves heterogeneous electronic devices which are connected in a local area network (LAN). The user activities are sensed by sensor and contents are recommended based on user's history, behavior, common data and etc. Scale of data storage and computing ability are needed for data analysis and data saving although the novel electronic devices are powerful. Cloud computing has recently emerged with the paradigm of IoT which becomes significant for data storage and data computing area [13]. The idea of cloud computing is that the computing is in the internet. In a cloud computing service, it can quickly adapt to change its capabilities and massive storage capacity for the service consumer's needs [14]. In the smart home, for the functionalities, context aware capabilities are needed. Voice detection sensor, distance detection sensor, camera and etc. are sensors which can detect activities of residents. In the sensor network, sensors are connected with central controller that has computing capability and storage capacity.

Figure 1 illustrates the multimedia services in the smart home. The figure shows the sensors collect information from users and send the information to the electronic devices such as TV, tablet and audio system through LAN. The electronic devices are connected with the internet through wide area network (WAN). Electronic devices can download multimedia contents from serves through WAN and otherwise also upload user data to the servers.

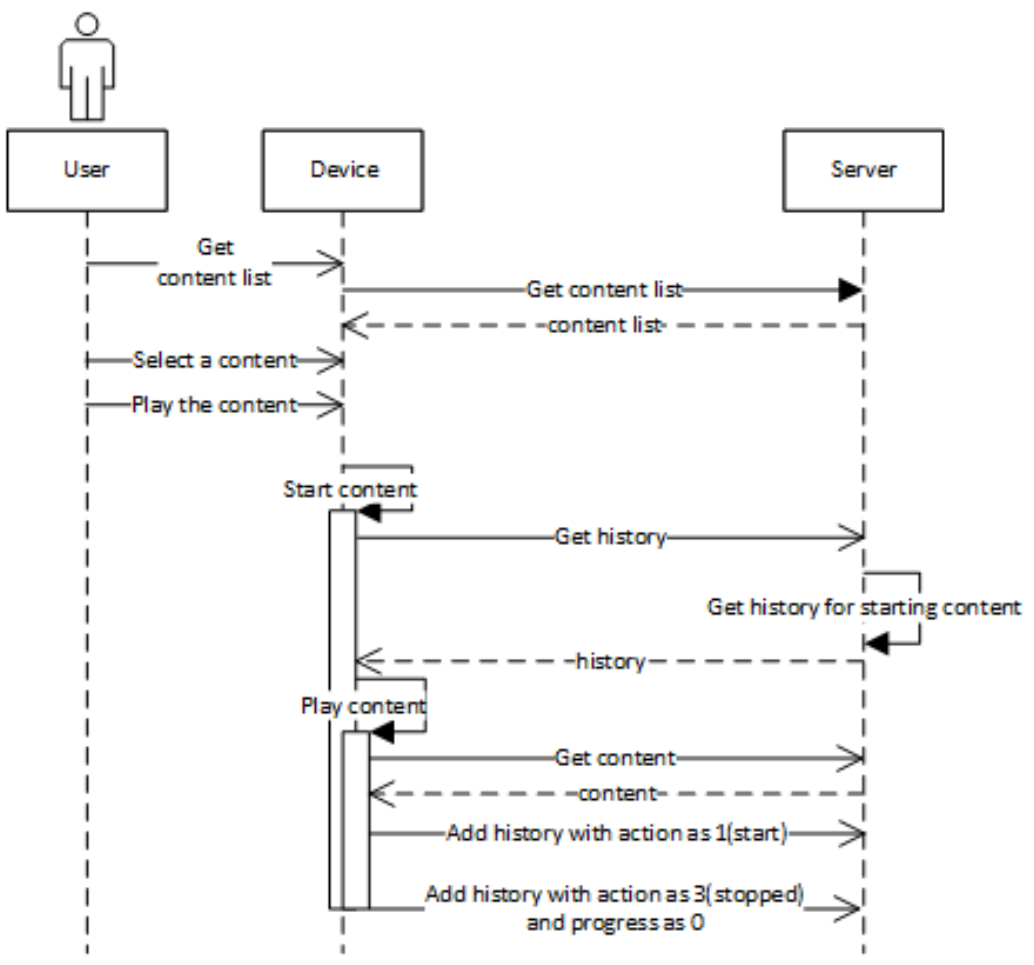

Figure 2. Sequence Diagram for Selecting Content to Play 


\section{Seamless Multimedia Service Mechanism for Personal Mobility}

Following sequence diagrams are designed for a multimedia services in the smart home which illustrate interaction between the user, device and server.

The user is a resident of the smart home and also the consumer of the multimedia service. The device is emulated as a pc application which includes console and information displays. Server is an application which provides web services for supporting conceptual services to the device emulator.

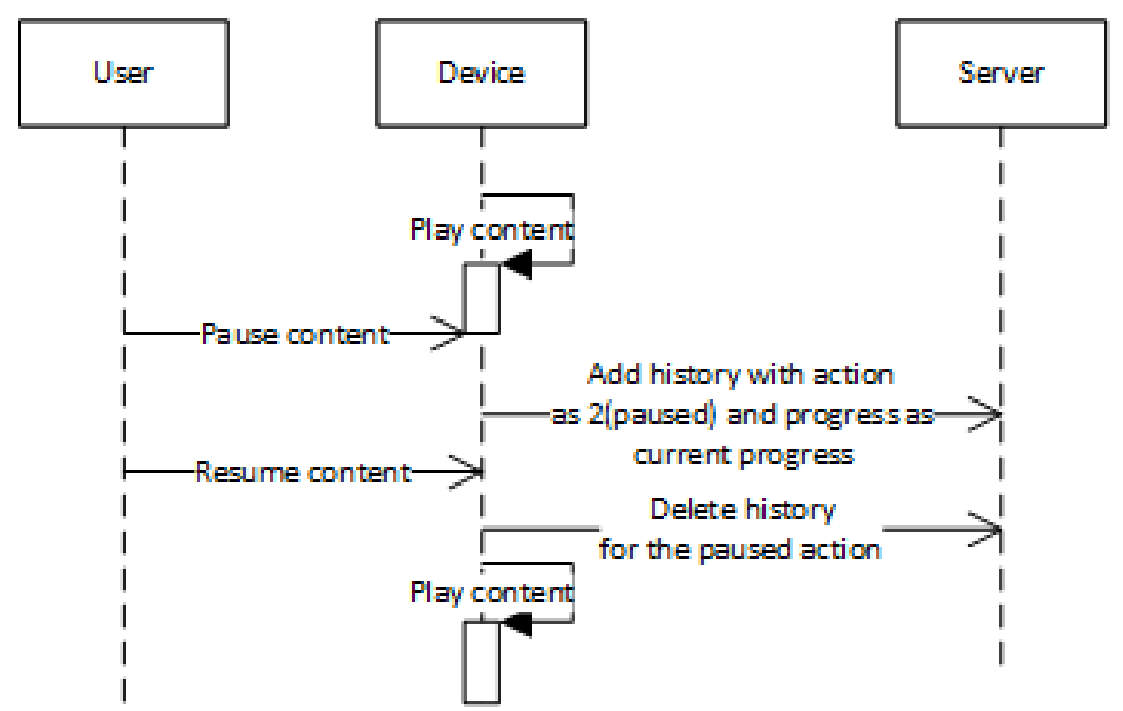

Figure 3. Sequence Diagram for Pausing and Resuming

Figure 2 shows when a user turns on a device, the user can request a content list from the server. Then a content is selected to play by the user. For starting the content, the device request the server for getting history data. The process is used for recommendation of continuing a previously paused or stopped content. After checking the recommendation, a content is played on the device. When a content is started to play, a history is saved, and a history is saved when a content is stopped.

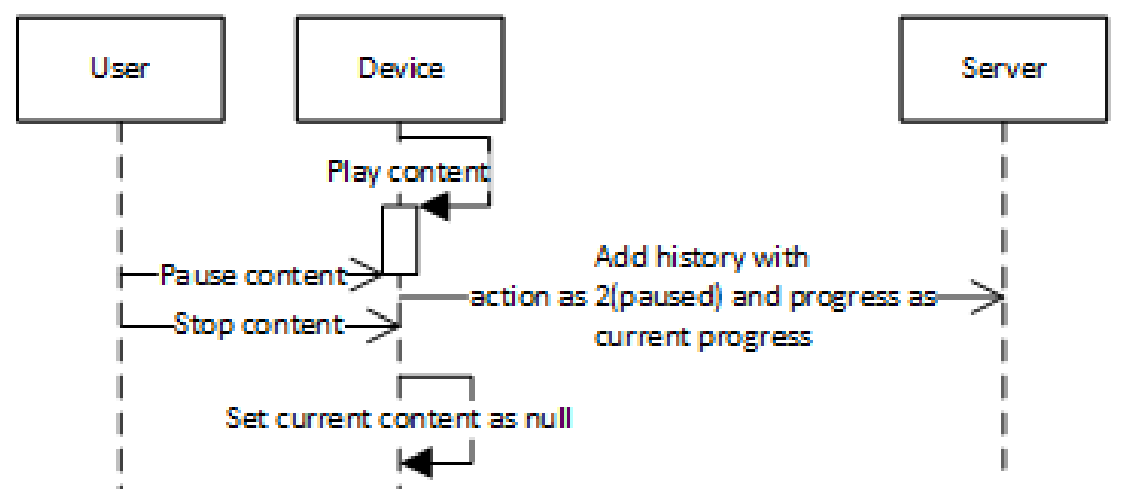

Figure 4. Sequence Diagram for Pausing and Stopping

Figure 3, 4 and 5 illustrate pausing, stopping and resuming situations when a user plays a contents through the multimedia services in the smart home. The multimedia contents are continuous data. Therefore, for some case it is needed to be paused, stopped and resumed. Those user activities are should be recorded as history data of a user. For deal with the situations, the figures show interactions of the elements of the Multimedia Service. 
Figure 3 shows a sequence that illustrates when a content is playing, then the content is paused and resumed by a user. When a content is paused by a use, then the device send a message for recording the action. The action is an explicit of the user in the smart home environment. In the situation, the status of user's action and content both are sent as parameters to the server. Later the information is used for recommendations. Moreover, resuming also considered in the sequence. The step deletes the paused information from the server and plays the content on the device.

Figure 4 considers a content is paused and stopped. The activity of pause and stop are needed to stop the content which plays on the device. Therefore, the step of stop activity is not needed to send message to server, but the process on the device is needed.

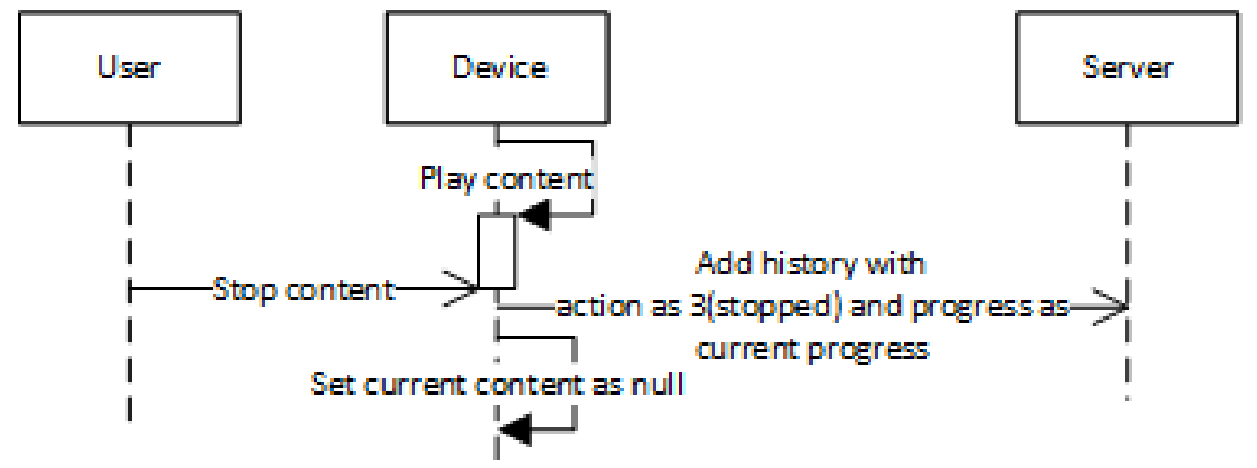

Figure 5. Sequence Diagram for Stopping

Figure 5 shows a situation that illustrates a content is stopped by a user. Then it also is needed to send message to the server for recording the information to be use for the recommendations.

\section{A Design of Seamless Multimedia Services Based on Content Profile Using User and Device ID}

Using the history data of users, the system can recommend some behaviors, e.g., if a user turn on with a paused content, then using the history data, the system should recommend to the user. But, may the user rejects the recommendation. Therefore, for smarter recommendation, more context of the user needs to be considered.

A home environment can be separated by several place. The user of multimedia services in the home environment, who may want the seamless multimedia service in the entire home. The smart home equips with IoT devices for multimedia which can support interoperability of devices in the Multimedia Service. The identifiers of users and devices are used for addressing the multimedia contents to the specific device of the specific user. The content profiles are data which are used for synchronizing the states of devices.

Figure 6 shows the scenario of multimedia services in the smart home. The initial state is the user plays multimedia contents on the device $\mathrm{A}$ in location A. The activity of the user with device $\mathrm{A}$ is up loaded to the service in the internet for synchronizing the information. The user can do some action on the Device A like play, pause and stop. In this process, the user's activity information and progress of the contents exist in the server which can be accessed by anywhere through internet. After some time, the user accesses a device in a location (same or other), the device in the location synchronizes the information from server. Then the user can continue the contents. In the figure, the user moves to location $\mathrm{B}$ where includes device $\mathrm{B}$. The user can continue the contents in the environment. 


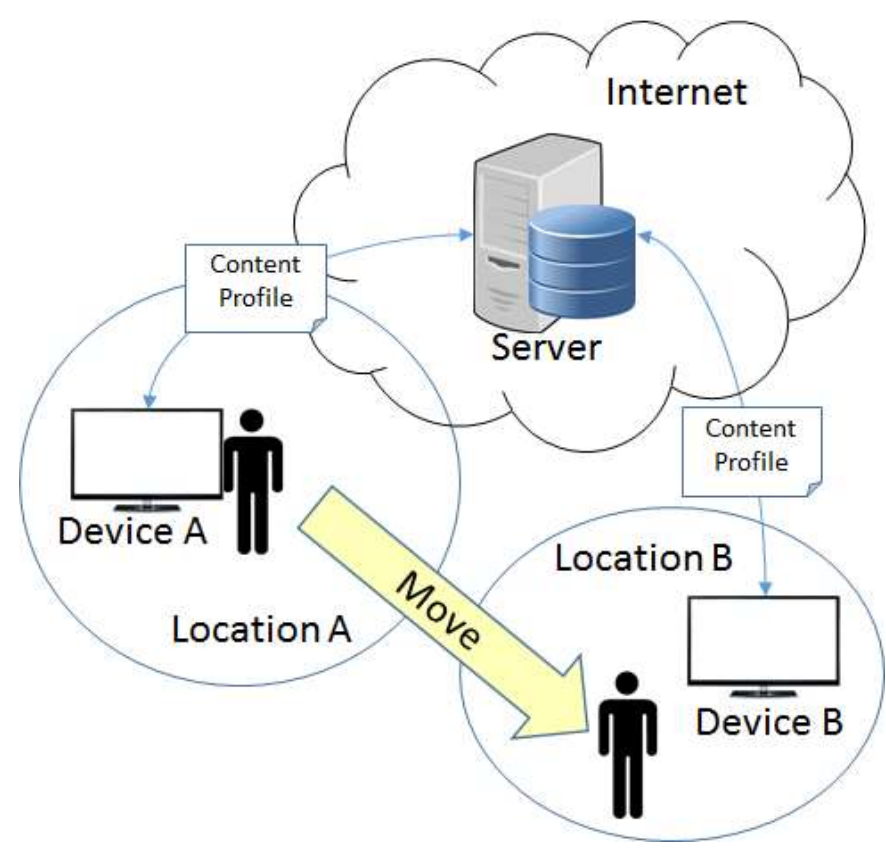

Figure 6. Scenario of Seamless Multimedia Services in Smart Home

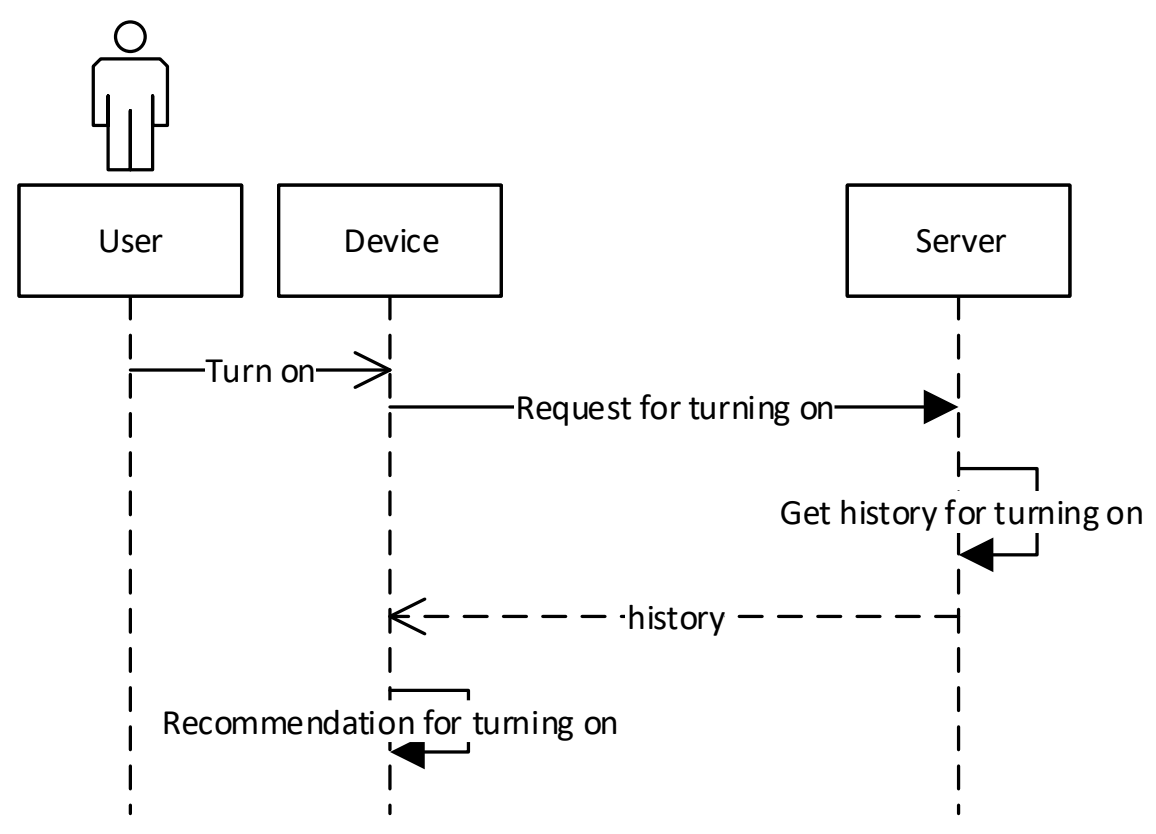

Figure 7. Sequence Diagram for the Device is turned on by the User

Figure 7 shows the process when a device is turned on by a user. The user turns on the device, then the device sends message to server for the situation. We assume various user data and common data are available for the situation of the user in the server. Then it needs higher computing capability and storage capacity in the server. Otherwise, as shown as the figure, the recommendation result can be processed in the device.

Figure 8 shows the flowchart for getting history of starting content. The process is run on the server for processing request from the device to return history data when a content is started to play by the user. In the diagram, history means the history information that includes user's action and progress of content. The parameter user_no is the identifier of the user and content_no is the identifier of the content. The server firstly get the paused 
history using user_no and content_no for the user. If the paused history is not exist in the $\mathrm{DB}$, then the stopped history is got. And when sopped history is null and the progress of the history is 0 , then set the history as null.

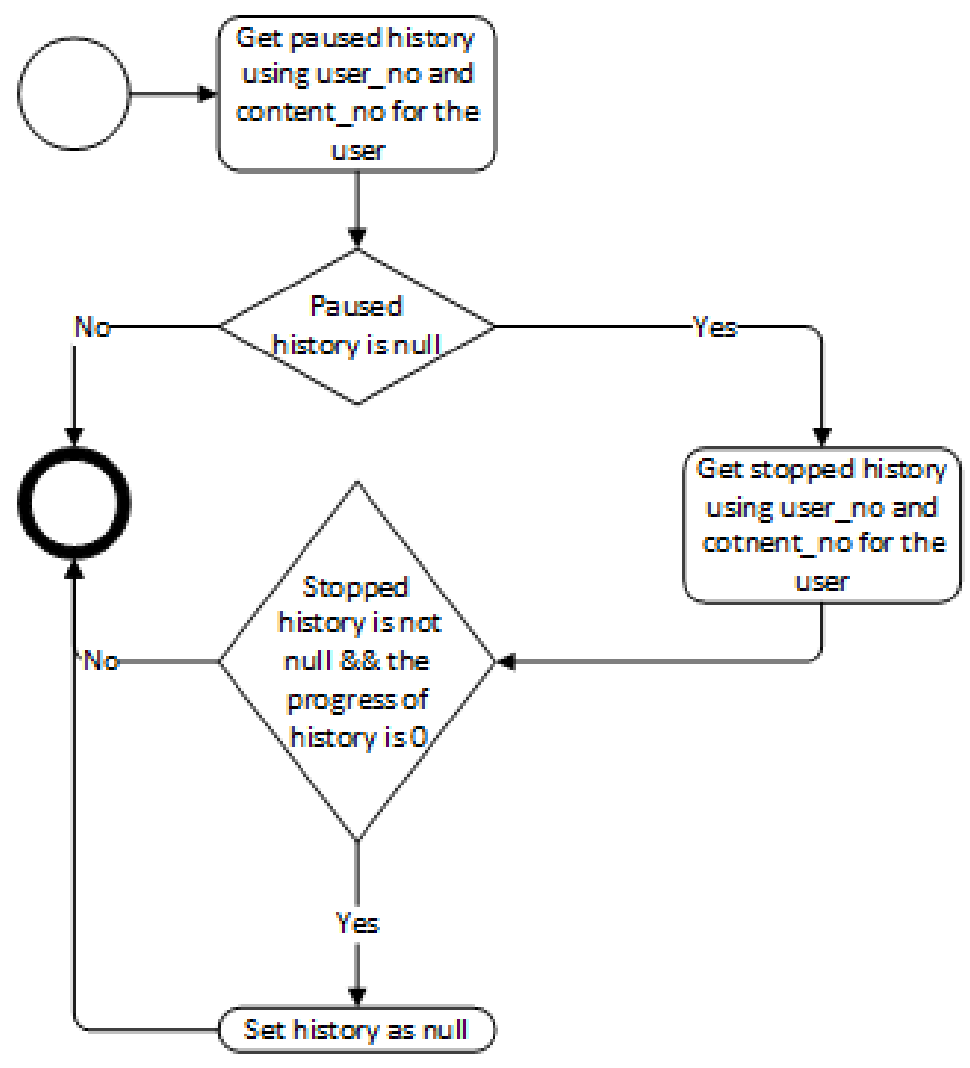

Figure 8. Flowchart for Getting History of Starting Content

Figure 9 shows the flowchart for starting content in the device. The process is run on the device for recommending a previously paused or stopped content to the user. When the device receives the history information is not null then it checks the action from history. The action value is 2 and 3, then the device suggest to the user for continuing the content. If the user agree with the suggestion then the content is played on the previously saved progress.

Figure 10 is an ER diagram of the Multimedia Service. The table "t_user" is used for saving the information of users which includes ID, password and other personal information. The table "t_history" is used for saving the history data of users. The activities of a user is importance data for data analysis. Those data also are used for recommendation functions. Table "t_content" and "t_device" are used for saving information of contents and devices. In the server, there are a lot of contents available. For a user may there more than one devices are registered. 


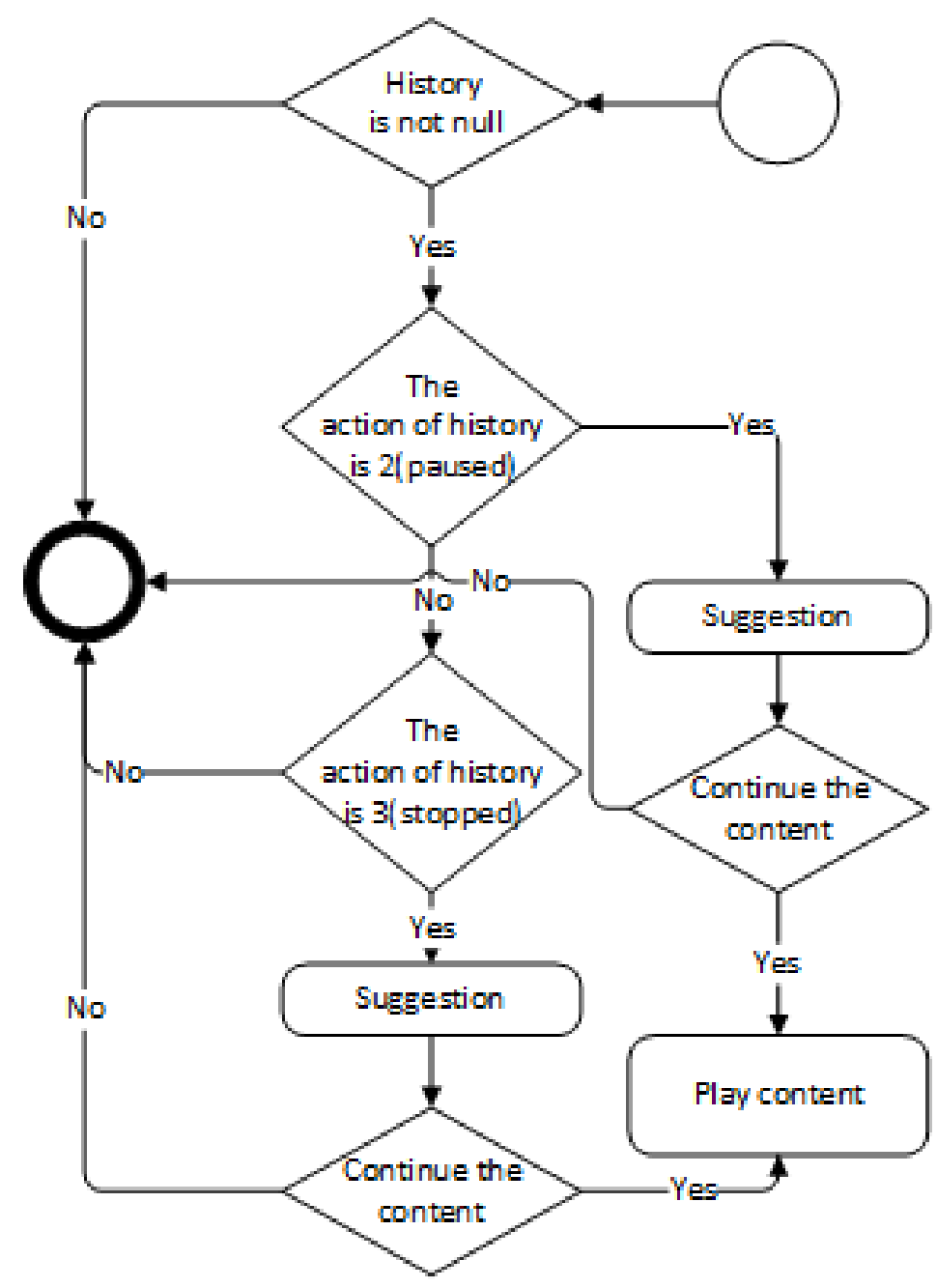

Figure 9. Flowchart for Starting Content in the Device

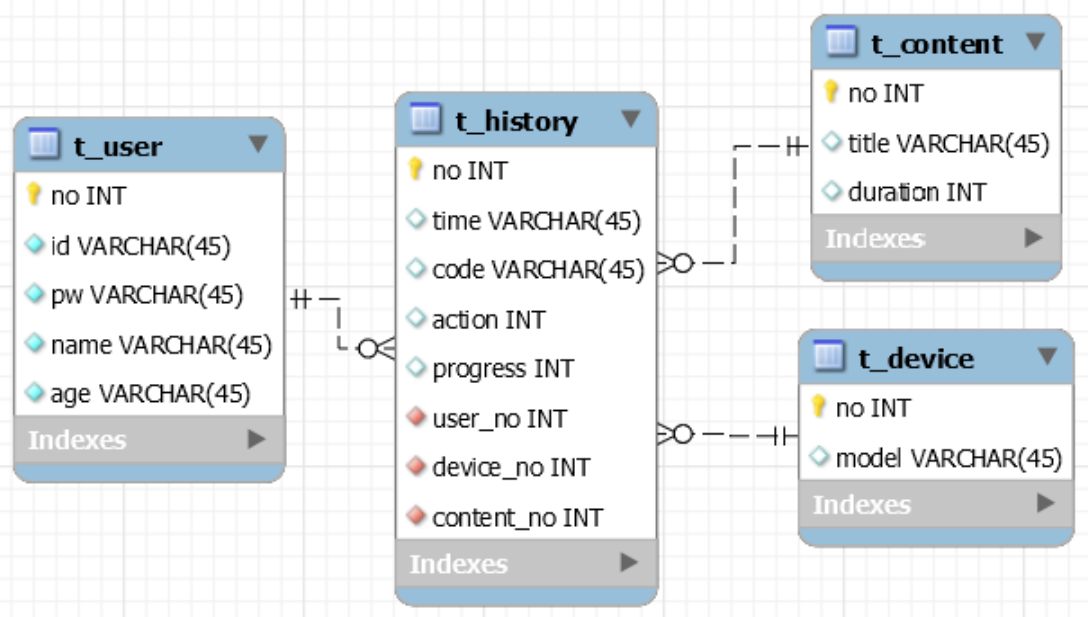

Figure 10. ER diagram of the Multimedia Service 


\section{Implementation of Seamless Multimedia Services}

Table 1 shows the environment of development for the Multimedia Service. The system have designed as server/client architecture. The client application is a windows application built in C\# with .NET framework. And the server application is also a windows application which supports WCF web services for communicating with the client application [15]. The database have been built by MySQL DBMS.

Table 1. Development Environment

\begin{tabular}{|l|l|}
\hline Operating system & Windows 7 Ultimate 64 bit \\
\hline Tool & $\begin{array}{l}\text { Visual Studio Community 2015, } \\
\text { MySQL Workbench 6.2 CE }\end{array}$ \\
\hline Framework \& Library & $\begin{array}{l}\text {.NET Framework 4.5.2, } \\
\text { Newtonsoft.Json }\end{array}$ \\
\hline DBMS & MySQL Community Server 5.7 \\
\hline
\end{tabular}

Figure 11 shows the server application's user interface. The application shows a list in the interface. When a client application connect to the server application then the information of user and device are shown in the list.

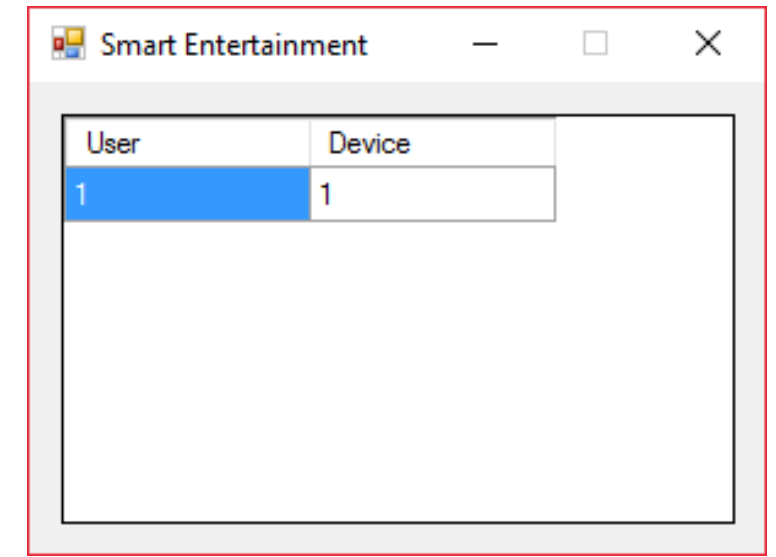

\section{Figure 11. Server Application for Logging User Activity}

Figure 12 shows the result of the client application with contents recommendation. The client application emulates a multimedia device. After the application is emulated to turn on, a display, content list and buttons are shown. The content list is used for showing recommended contents.

Figure 13 shows when the client application is turned on then the activity of user is recommended. The recommendation is depended on the user's data which involves user's activity history data and service consumption history data. 


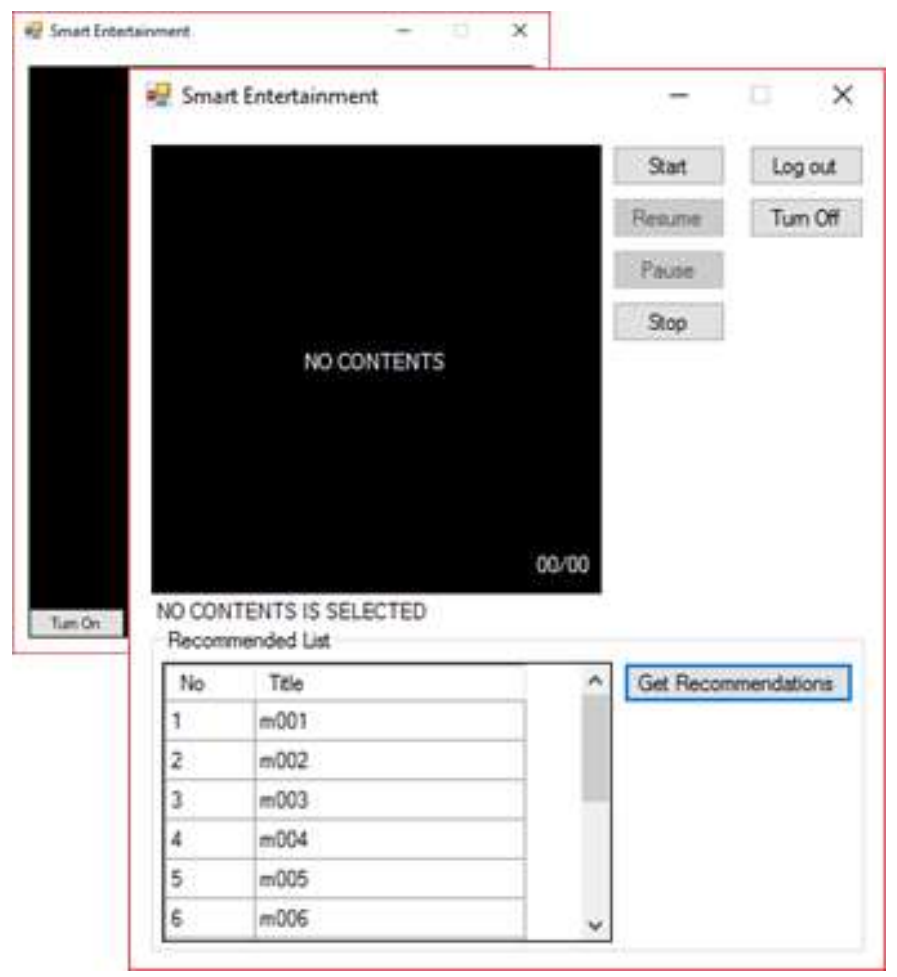

Figure 12. Client Application with Contents Recommendation

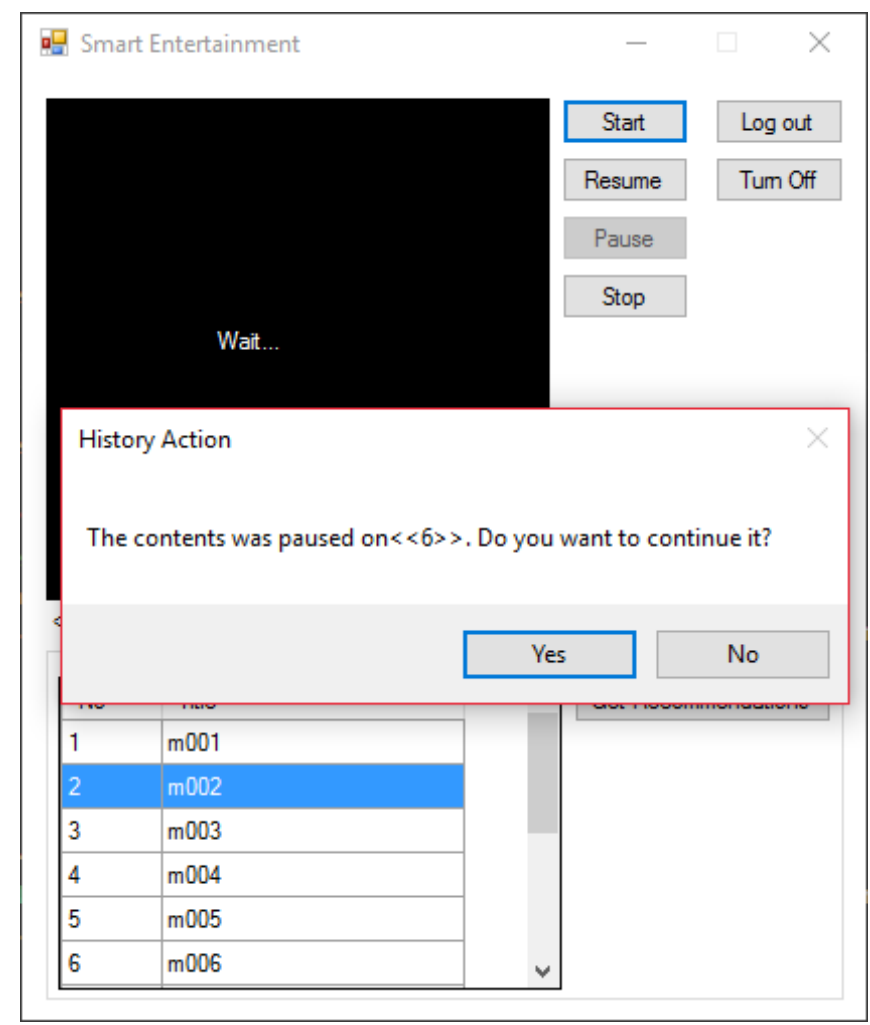

Figure 13. Client Application with Activity Recommendation

\section{Conclusions}

In this study, we presented the seamless multimedia service mechanism in the smart home. In order to support seamless multimedia services in smart homes, the users, 
devices, locations and contents are needed to be considered. The proposed mechanism considers multi-spaces, multi-devices and multi-users for mobility of users in the smart home. For the mechanism, the user and device ID are used to recognize the user and device in the smart home system. With the identifiers of the user and device, the user and device can be tracked and verified in the system. The content profile is used to synchronize the content by the interoperability with the server. Based on the analysis and consideration, the solution is proposed and the prototype application is developed to demonstrate the mechanism of the multimedia services for the user mobility in the smart home.

\section{Acknowledgments}

This work was supported by Institute for Information \& communications Technology Promotion (IITP) grant funded by the Korea government (MSIT) (No.2017-0-00756, Development of interoperability and management technology of IoT system with heterogeneous ID mechanism), and this research was supported by the MSIT (Ministry of Science and ICT), Korea, under the ITRC (Information Technology Research Center) support program (IITP-2017-2014-0-00743) supervised by the IITP (Institute for Information \& communications Technology Promotion), Any correspondence related to this paper should be addressed to DoHyeun Kim.

\section{References}

[1] K. Dey, "Understanding and using context", Pers. Ubiquitous Comput., vol. 5, no. 1, (2001), pp. 4-7.

[2] S. Ali and D. H Kim, "Effective and comfortable power control model using Kalman filter for building energy management", Journal of Wireless Personal Communication, vol. 73, no 4, (2013), pp. 14391453.

[3] G. A. Dávi, E. Caamaño-Martín, R. Rüther and J. Solano, "Energy performance evaluation of a net plusenergy residential building with grid-connected photovoltaic system in Brazil", Energy and Buildings, vol. 120, (2016), pp.19-29.

[4] R. Ahmad, S. Ali and D. H. Kim, "A Multi-Agent System for Documents Classification”, 2012 International Conference on Open Source Systems and Technologies (ICOSST), (2012).

[5] S. Sadiq, Y. Yan, M.-L. Shyu, S.-C. Chen and H. Ishwaran, "Enhancing Multimedia Imbalanced Concept Detection Using VIMP in Random Forests", In Information Reuse and Integration (IRI), 2016 IEEE 17th International Conference on, (2016), pp. 601-608.

[6] F.-L. Huang and S.-Y. Tseng, "Predictable smart home system integrated with heterogeneous network and cloud computing", In Machine Learning and Cybernetics (ICMLC), 2016 International Conference on, vol. 2, (2016), pp. 649-653.

[7] H. Gu, Y. Diao, W. Liu and X. Zhang, "The Design of Smart Home Platform Based on Cloud Computing", International Conference on Electronic \& Mechanical Engineering and Information Technology, (2011).

[8] X.Ye and J. Huang, "A framework for Cloud-based Smart Home", Computer Science and Network Technology (ICCSNT), 2011 International Conference on, (2011).

[9] S. Chenishkian, "Building Smart Services for Smart Home", in Proceedings of the IEEE 4th International Workshop on Network Appliances, (2002), pp. 215-224.

[10] J. H. Choi, D. K. Shin and D. G. Shin, "Research and Implementation of the Context-Aware Middleware for Controlling Home Appliances", IEEE Transactions on Consumer Electronics, vol. 51, no. 1, (2005), pp. 301-306.

[11] Y. Isoda, S. Kurakake and H. Nakano, "Ubiquitous Sensors based Human Behavior Modeling and Recognition using a patio-Temporal Representation of User States", in proceedings of 18th International Conference on Advanced Information Networking and Applications (AINA '04), (2004), pp. 512-517.

[12] F. Rasheed, Y. K. Lee and S. Lee, "Towards Using Data Aggregation Techniques in Ubiquitous Computing Environments", in Proceedings of the 4th Annual IEEE International Conference on Pervasive Computing and Communications Workshops, (2006), pp. 369-372.

[13] M. Deng, M. Petkovic, M. Nalin and L. Baroni, "A home healthcare system in the cloud - addressing security and privacy challenges", IEEE 4th International Conference on Cloud Computing, (2011).

[14] Y. H. Zhang, J. Zhang and W.H. Zhang, "Discussion Of A Smart House Solution basing Cloud Computing", International Conference on Communications and Intelligence Information Security, (2010).

[15] "What Is Windows Communication Foundation", <https://msdn.microsoft.com/enus/library/ms731082(v=vs.110).aspx>, (2017). 


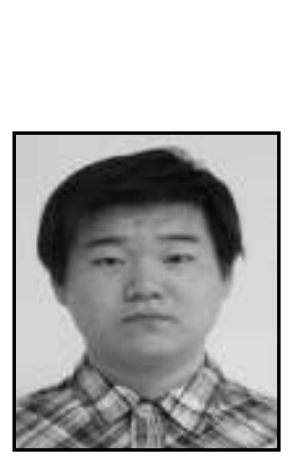

\begin{abstract}
Authors
Wenquan Jin, he received the B.S. degree in computer science and technology from Yanbian University of Science and Technology, China, in 2013, and the M.S. degrees in computer engineering from the Jeju National University, Korea, in 2015. Now he is a Ph.D candidate in the Jeju National University, Korea. His research interests include sensor networks, M2M/IOT, IoT intelligent service, and mobile computing.
\end{abstract}

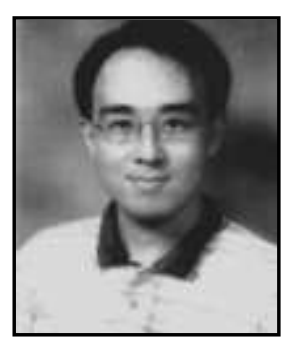

Do-Hyeun Kim, he received the B.S. degree in electronics engineering from the Kyungpook National University, Korea, in 1988, and the M.S. and Ph.D. degrees in information telecommunication the Kyungpook National University, Korea, in 1990 and 2000, respectively. He joined the Agency of Defense Development (ADD), from Match 1990 to April 1995. Since 2004, he has been with the Jeju National University, Korea, where he is currently a Professor of Department of Computer Engineering. From 2008 to 2009, he has been at the Queensland University of Technology, Australia, as a visiting researcher. His research interests include sensor networks, $\mathrm{M} 2 \mathrm{M} / \mathrm{IOT}$, energy optimization and prediction, intelligent service, and mobile computing. 